\title{
Immunity and tolerance to infections in experimental
}

\section{hematopoietic transplantation}

Agostinho Carvalho ${ }^{\mathrm{a}, \mathrm{b}}$, Cristina Cunha ${ }^{\text {a }}$ and Luigina Romani ${ }^{\mathrm{a},{ }^{*}}$

${ }^{a}$ Microbiology Section, Department of Experimental Medicine and Biochemical Sciences, University of Perugia, Perugia, Italy

${ }^{\mathrm{b}}$ Life and Health Sciences Research Institute (ICVS), School of Health Sciences, University of Minho, Braga, Portugal

* Corresponding author: Luigina Romani, Microbiology Section, Department of Experimental Medicine and Biochemical Sciences, University of Perugia, Via del Giochetto, 06126 Perugia, Italy. Ph and Fax:+39075 5857411. E-mail: 1romani@unipg.it 


\begin{abstract}
Resistance and tolerance are two types of host defense mechanisms that increase fitness in response to fungi. Several genetic polymorphisms in pattern recognition receptors, most remarkably Toll-like receptors (TLRs), have been described to influence resistance and tolerance to aspergillosis in distinct clinical settings. TLRs on dendritic cells pivotally contribute in determining the balance between immunopathology and protective immunity to the fungus. Epithelial cells also contribute to this balance via selected TLRs converging on indoleamine-2,3-dioxygenase (IDO). Studies in experimental hematopoietic transplantation confirmed the dichotomy of pathways leading to resistance and tolerance to the fungus providing new insights on the relative contribution of the hematopoietic/nonhematopoietic compartments.
\end{abstract}


Hematopoietic stem cell transplantation (HSCT) has been proven an effective therapeutic strategy for hematological malignancies and immune disorders. Despite the significant advances witnessed in the technique of transplantation and in therapies to avoid rejection episodes, this practice is nonetheless prone to the harmful consequences of the nonspecific immunosuppression required for survival of the allograft. In order to minimize these severe side effects, research is focusing on the exploitation of therapies leading to tolerance induction and immune homeostasis.

Fungal infections remain nowadays a major cause of transplant-related morbidity and mortality in the HSCT setting [1]. One of the most important risk factors for fungal infections in HSCT has historically been neutropenia. However, a predominance of aspergillosis cases occurring in the post-engraftment rather than the neutropenic period in transplant recipients has been described [2]. This suggests that host immunity is crucial in eradicating infection, but an overzealous immune recovery can also be harmful and may contribute towards worsening disease. Therefore, proper immunotherapies restraining deregulated innate and adaptive responses could provide control of immune overreactions in fungal infections [3].

In this review, we shall consider the interplay between recipient- and donor-dependent mechanisms of protection that are coordinately induced for optimal anti-microbial resistance with minimum histopathology (otherwise referred to as protective tolerance). In experimental candidiasis and aspergillosis, the balance between Th1 cells (that provide antifungal resistance) and regulatory $\mathrm{T}$ cells (Tregs) that limit the consequences of the associated inflammatory pathology may provide the basis for the occurrence of functionally distinct modules of immunity for resistance and tolerance [4]. Specifically, we will discuss the role of these protective mechanisms in antifungal immunity and how these can be successfully exploited to elicit antimicrobial immunity and concomitant tolerance. Resistance and 
tolerance are two types of host defense mechanisms that increase fitness in response to fungi [4].

\section{Inflammation and immunity in fungal infections: the current view}

The current understanding of the pathophysiology underlying fungal infection and disease fits with the bipolar nature of the inflammatory process in infection $[3,5]$. In order to achieve the best-fitted form of antifungal resistance, innate responses interplay with adaptive immunity, preserving the precarious balance between the opposing effects of inflammation on infection. Inflammation is in part required for protection - particularly in mucosal tissues during the transitional response occurring temporally in the bridging between innate and adaptive responses. However, uncontrolled inflammation may eventually aggravate disease and ultimately oppose pathogen eradication. In this context, taming overzealous and exaggerated inflammatory responses has been shown to rely on indoleamine 2,3-dioxygenase (IDO), a rate-limiting enzyme in the tryptophan catabolism involved in the inhibition of cell proliferation, including that of potentially harmful activated $\mathrm{T}$ cells [6], tryptophan catabolites and Treg function. In contrast, IL-23 and the Th17 pathway, by down-regulating tryptophan catabolism, may instead favor pathology and serve to accommodate the apparently paradoxical association of chronic inflammation with fungal persistence [7]. It is of interest that the IL-23/Th17 pathway has been recently acknowledged to play an important role in transplant rejection and tolerance [8]. In this regard, strategies antagonizing IL-23/IL-17, including the administration of synthetic kynurenines, could represent a means of harnessing progressive or potentially harmful inflammation $[3,5]$. Similarly, we have recently found that blocking inflammatory pathways in vivo could also be exploited for the development of siRNA therapeutics to attenuate inflammation in aspergillosis [9]. 
The above findings may assist in explaining the fact that, despite humans being constantly exposed to fungi, fungal diseases are relatively rare. This implies that the continuous coexistence between fungi and their mammalian hosts plays a underrated contribution to the plasticity of the immune system. In fact, recent evidence supports that the unremitting integration of pro- and anti-inflammatory signals in response to fungi is critical for a proper control of infection and T-cell homeostasis. In this context, IDO and tryptophan catabolites are major determinants of this delicate balance, by providing the host with immune mechanisms adequate for protection without necessarily eliminating fungal pathogens which would impair immune memory - or causing unacceptable levels of tissue damage [3, 5]. Ultimately, the mechanism used by the immune system for suppressing autoreactive responses could conceivably be employed for therapeutic purposes in transplantation.

\section{Resistance and tolerance to fungi in HSCT}

\section{Dendritic cells provide antifungal immune resistance and tolerance}

Dendritic cells (DCs) orchestrate the adaptive immune responses to Aspergillus [9-10]. The plasticity of fungus-pulsed DCs in initiating disparate Th cell responses largely relies upon the specialization and cooperation between distinct DC subsets and the discriminative recognition of fungal morphotypes by diverse innate recognition receptors [11-13]. For this reason, DCs are central in the early decision mechanisms defining immune responses to fungi, determining resistance or susceptibility to infection upon the host/fungus interaction.

Type I IFN-producing plasmacytoid DCs (pDCs) define Th responses and are able to induce and sustain protective tolerance, by supporting the generation of Treg with suppressive activity [14]. Expansion of pDCs (FL-DCs) is contingent upon the hematopoietic growth factor FLT3L and not GM-CSF/IL-4, known to expand conventional, myeloid DCs (GM- 
DCs) [15]. A comparative analysis of murine and human DC subsets for Th priming against Aspergillus revealed that FL-DCs, but not the conventional GM-DCs, conferred resistance to aspergillosis in experimental HSCT [13]. Interestingly, GM-DCs, although capable of an efficient control of fungal growth, were nevertheless associated with inflammatory overreactions. These findings highlight the notion that immunotherapy with fungus-pulsed DCs may generate both protective and non-protective antifungal responses, corroborating the importance of DC plasticity and functional specialization in response to fungi [16]. The infusion of selected DC subsets along with donor T cells further enlightens the beneficial or detrimental potential of each DC subpopulation in HSCT. In this regard, whereas the infusion of GM-DCs greatly accelerated graft- $v s$. -host-disease (GVHD) onset by T cells, FL-DCs not only failed to do so but actually totally prevented it [13]. In fact, FL-DCs were able to control inflammation both in infection and GVHD, a feature associated with the concomitant activation of IFN $-\gamma^{+}$Th1 cells and FOXP $3^{+} \mathrm{IL}-10^{+} \mathrm{CD} 25^{+}$Tregs [13].

Vaccination is a potentially important strategy for reducing the risk of infections after HSCT. An improved understanding of the pathogen/DC interaction may allow the potential use of pathogen- or TLR-conditioned DCs for the induction of patient-tailored Tregs with indirect anti-donor allospecificity. In this regard, we have recently found that different cell wall components of Aspergillus are endowed with the capacity to activate distinct Th cell responses [17]. Most importantly, some fungal antigens retained their immunodominant Th1/Treg activating potential across mice and humans, suggesting that the broadness and specificity of the human T-cell repertoire against the fungus could be selectively exploited with defined immunoactive fungal antigens.

It has been demonstrated that a division of labor occurs between functionally distinct Tregs that are coordinately activated by a CD28/B7-dependent costimulatory pathway after 
exposure of mice to Aspergillus resting conidia [18]. Inflammation is controlled by the expansion, activation and recruitment of $\mathrm{CD} 4{ }^{+} \mathrm{CD} 25^{+}$Tregs that suppress neutrophils through the combined actions of IL-10 and CTLA-4. Late in infection, and similarly in allergy, tolerogenic Tregs, which produce IL-10 and TGF- $\beta$, inhibit Th2 cells and prevent allergy to the fungus. Thus, the capacity of Tregs to inhibit innate and adaptive immunity traits is crucial to their regulatory role in fungal infections [13]. Altogether, these findings suggest that FL-DCs are fully competent at inducing antifungal resistance and protective tolerance upon adoptive transfer in HSCT recipients through the combined action of immunizing and tolerizing DCs. In contrast, GM-DCs are capable of immunotoxicity, which may include the promotion of inflammation and GVHD.

\section{The nonhematopoietic compartment contributes to protective tolerance to Aspergillus}

Airway epithelial cells (ECs) are no longer considered innocent bystanders, being now recognized as central participants in innate and adaptive immune responses as well as mucosal inflammation and allergy [19]. Through the activation of immune receptors, ECs may determine the balance between a state of "mucosal homeostasis", required for optimal organ function, and "mucosal injury," leading to mucosal inflammation and barrier breakdown. However, recent evidence has also indicated ECs as key players in immunity to respiratory pathogens such as Mycobacterium tuberculosis via an IFN- $\gamma /$ IDO axis culminating in the inhibition of Th17 cell responses [20].

Airway ECs have been traditionally regarded as reservoirs allowing for the immune evasion of the fungus due to the ability of ingested conidia to survive inside them [21]. However, recent reports have also established respiratory ECs as key contributors to host 
defense against $A$. fumigatus [22-24]. It is well established that the proper control of infection and associated inflammatory reactions require IDO induction and consequent production of tryptophan metabolites with immune-regulatory activities, contributing to the maintenance of the Treg/Th17 balance [5, 7]. As clearly shown in susceptible mice, Tregs and Th17producing cells mediate antagonizing roles in aspergillosis, where increasing levels of IL-17driven inflammation occurred alongside decreased anti-inflammatory Treg responses, resulting in inflammatory overreactions [7]. A reciprocal antagonistic relationship was also found between IDO and the Th17 pathway, with IDO restraining Th17 responses $[5,25]$ and IL-17A inhibiting IDO [25]. Accordingly, IDO overexpression in airway ECs was found to restrain $\mathrm{CD}^{+} \mathrm{T}$ cell activation to the fungus, an activity that was nevertheless dispensable in the presence of IDO-expressing tolerogenic DCs [26]. Indeed, in experimental HSCT, IDOcompetent DCs provided protective tolerance to the fungus, by crucially affecting the balance between immunity and tolerance in the lung [13]. These data are consistent with the role of IDO in hematology, its requirement for the generation of Tregs and for protection against GVHD [6].

We have recently found that ECs contribute to antifungal immunity by providing protective tolerance through a TLR3/TRIF-dependent pathway converging on IDO and may compensate for the lack of IDO on hematopoietic cells. [24]. ECs activate IDO via TLR3, that is abundantly expressed both intracellularly and on the cell surface of ECs [27], and IFN- $\gamma$ to which ECs also respond. The failure to activate IDO likely accounted for the high levels of inflammation seen in Trif $^{/-}$mice, as proved by the immunorestorative properties of exogenously administered kynurenines, known to induce apoptosis of IL-17A $\mathrm{A}^{+} \gamma \delta^{+}$cells [7]. We have shown that exogenous administration of kynurenines restored protective tolerance to the fungus [7]. Thus, the therapeutic effects of kynurenines may also encompass IDO deficiency in ECs to induce Treg expansion, [19, 28], restrain $\mathrm{CD}^{+}{ }^{+}$-cell proliferation [7], 
and produce IL-10 [24]. As a matter of fact, the highly polarized Th2/Th17 responses seen in Trif $^{/-}$mice are immunological features compatible with the functional program activated in lung DCs by the TRIF pathway [9]. Interestingly, the TLR3 ligand poly(I:C) has also a direct costimulatory effect on IFN $-\gamma^{+} \gamma \delta^{+}$cells, a finding suggesting that the protective effect of the TLR3/TRIF pathway in infection could be achieved through multiple mechanisms. Overall, our data shed light on pathways of immune resistance and tolerance to the fungus that likely take place in a hematopoietic transplantation setting. It appears that protective tolerance to the fungus is achieved through a TLR3/TRIF-dependent pathway activating Th1/Treg cells via IDO expressed on both the hematopoietic/nonhematopoietic compartments. The MyD88 pathway instead likely provides antifungal resistance, i.e. the ability to restrict the fungal growth, likely through defensins and other effector mechanisms. However, the ability of mice to clear the fungus in the relative absence of the MyD88 pathway [11,29], clearly indicates redundancies and hierarchy in antifungal mechanisms of resistance. Ultimately, the finding that both Candida albicans [30] and A. fumigatus, two major human fungal pathogens, exploit the TRIF-dependent pathway at the interface with the mammalian hosts, indicate that the combinatorial configuration of antifungal resistance and tolerance is an advantageous option.

\section{Genetic susceptibility to fungal infections in HSCT}

Given the significance of innate immune receptors, namely TLRs, in the eliciting of immune responses, it is not surprising that genetic variability of these receptors may affect their function and may account, in part, for the inherited differences in susceptibility to infection [31] (Table 1). Most genetic studies performed until recently have focused on the highly polymorphic TLR4 gene, in which the presence of two co-segregated polymorphisms D299G and T399I - has been found to compromise the ligand-binding properties of TLR4 
[32]. These polymorphisms have been linked with blunted airway responses to inhaled LPS in human individuals and attenuated LPS-induced responses in primary airway ECs.

One of the first studies establishing a relationship between TLR4 polymorphisms and aspergillosis described an increased susceptibility of D299G carriers to a chronic form of pulmonary aspergillosis [33]. Shortly thereafter, the same TLR4 polymorphism was also found to increase susceptibility to invasive aspergillosis (IA) in HSCT recipients from unrelated donors, an observation validated in two different cohorts of patients [34]. Interestingly, a previous study, though modest in sample size, had failed to associate the same donor polymorphism with IA in HSCT recipients [35]. These apparently discrepant findings further highlight the intricacies of genetic associations with infectious complications in the transplantation setting, which may ultimately depend on the type of transplant and associated clinical variables. In this regard, we have recently described an association between donor D299G and colonization by A. fumigatus, but not invasive disease, in a cohort of T-celldepleted transplant recipients from related donors [36]. This suggests that, despite its prognostic potential, the presence of D299G may not definitely predict the clinical transition from fungal colonization to invasive disease, at least in this particular transplant setting.

Since the abnormal TLR4 ligand-binding domain may be impairing fungal recognition, eventually contributing to fungal escape from immune surveillance, individuals harboring the D299G polymorphism are likely more prone to develop fungal colonization following transplant. However, TLR4 polymorphisms have also been shown to protect from hyper-inflammatory diseases, including atherosclerosis and related conditions [37]. Therefore, predisposition to fungal colonization may be compensated by the lack on an overreactive inflammatory response, which may ultimately condition pathogen elimination. In this regard, exaggerated inflammatory responses, more than the fungus itself, may determine 
susceptibility to aspergillosis and other fungal infections [3]. Thus, and although functional studies are ultimately required to confirm this hypothesis, by limiting an exacerbated inflammatory response to the fungus, the D299G polymorphism could contribute to resistance to aspergillosis, despite evidence of fungal growth.

Recently, polymorphisms affecting pattern recognition receptors other than TLRs, in particular dectin-1, have also been addressed as potential predictive factors for the incidence of fungal diseases. Besides associating with recurrent mucocutaneous fungal infections [38], the dectin-1 Y238X polymorphism was also found to contribute to increased Candida colonization after HSCT [39]. In this context, we have recently found that the Y238X polymorphism contributed as well to increased susceptibility to aspergillosis in HSCT [23]. Moreover, the genetic association relied on both recipient and donor genetic backgrounds, with the highest risk being observed when polymorphic recipient/donor pairs were involved. The Y238X polymorphism generates a truncated protein unable to target the membrane and mediate $\beta$-glucan recognition, therefore resulting in defective cytokine production upon receptor engagement [38-39]. Although dectin-1 has been regarded as one major innate receptor leading to Th17 activation in response to Aspergillus [40], and the Y238X polymorphism was associated with impaired IL-17 production in response to C. albicans or $\beta$ glucan [38], we found that IFN- $\gamma$ and IL-10, in addition to IL-1 $\beta$, IL-6 and IL-17A, productions by human mononuclear cells carrying the Y238X polymorphism were defective upon $\beta$-glucan- or Aspergillus-specific stimulation. Thus, these findings point to a previously unsuspected role for dectin-1 in antifungal immunity, that is the ability to modulate immunity and tolerance via IFN- $\gamma / \mathrm{IL}-10$ production, both cytokines reflecting the activation of protective Th1/Treg antifungal responses. The contribution of recipient dectin-1 deficiency to the high risk of infection also reflects the crucial role of dectin-1 expressed on nonhematopoietic cells in the induction of immune protection to the fungus, highlighting the 
distinct, yet complementary, mechanisms of immune resistance and tolerance that are dependent on the hematopoietic/nonhematopoietic compartmentalization.

By influencing immune responses and inflammation, polymorphisms in cytokine genes may also help predict post-HSCT complications, including those of infectious nature. In this regard, we have recently found that polymorphisms in the IL-23/Th17 pathway may also define susceptibility to IA following HSCT [41]. Specifically, donor R381Q in the IL23R gene was associated with strong protection from IA, whereas R381Q in patients was correlated with improved overall survival [41]. These findings, although requiring replication in larger independent data sets, are consistent with the protective effects of IL-23 signaling attenuation in experimental aspergillosis [25]. The R381Q polymorphism has already been shown to confer protection against GVHD in recipients of grafts from HLA-identical donors [42]. Although the genetic findings have been thoroughly replicated, not much is known about the functional consequences of this variant. A recent study demonstrated that the protective effects displayed by the R381Q polymorphism were due to a deficient activation of IL-23-driven Th17 responses [43]. Interestingly, several polymorphisms in the ILI7A and $I L 17 F$ genes were not associated with susceptibility to IA in HSCT recipients [41], suggesting that additional studies are needed to define cellular and molecular mechanisms by which protection from aspergillosis is conferred by the disruption of the IL-23/Th17 axis.

\section{Immunotherapy in HSCT: what does the future hold?}

The new discoveries in the field of fungal immunology have offered new grounds for a better comprehension of cells and immune pathways that are amenable to manipulation in patients with or at risk of fungal infections, specifically those undergoing HSCT. Therefore, devising new strategies to expand and modulate the functions of distinct DC subsets 
associated with specific regulation of host immunity may provide novel immune-based therapies for use in HSCT. One potential difficulty associated with a DC-based treatment could be the control of the immunostimulatory/tolerogenic commitment. This could eventually be overcome by the targeting of DCs in vivo by biologic or pharmacologic agents. In this regard, thymosin- $\alpha 1$ appears to be a suitable candidate to modulate DC functioning for active and negative vaccination in transplantation. The therapeutic usage of IDO-mediated immune regulation has also been regarded as a promising strategy for use in transplantation. As a systemic enhancement of IDO activity by pharmacologic IDO induction would carry the risk of inducing general immunosuppression and IDO activity itself is modulated by the microenvironment and factors that cannot be controlled in vivo, an alternative therapeutic approach as a means of facilitating the generation of tolerance could be the enrichment of IDO activity at targeted sites, for example with IDO-competent DCs. In addition to these, further understanding of the role of ECs in the maintenance of immune tolerance could also be exploited as means of enhancing immunity against fungal pathogens. Specifically, taking advantage of the ability of ECs to up-regulate antimicrobial peptides endowed with antifungal activity could be regarded as promising target with potential application in the transplantation setting.

Finally, although the dissection of complex genetic traits modulating susceptibility to fungal infections is intricate, the contribution of host genetics may hold the key to expose new risk factors for these diseases. Therefore, new conceptual advances on the knowledge of host immunity need to be accommodated also from an immunogenetic point of view. Identifying the cellular and molecular bases affected by genetic polymorphisms may prove a very powerful research tool, allowing the identification of potential therapeutic targets and the design of prophylactic strategies exerting control over the outcome of immune pathways. The genetic screening of at-risk patients may ultimately be used to individualize treatments 
through the formulation of new targeted and patient-tailored antifungal therapeutics, likely improving the management and outcome of fungal infections.

\section{Acknowledgements}

We thank Cristina Massi Benedetti for digital art and editing. This work was supported by the Specific Targeted Research Project SYBARIS (LSHE-CT-2006), contract number 037899 (FP7), and by the Italian Project PRIN 2007KLCKP8_004. Agostinho Carvalho and Cristina Cunha were financially supported by fellowships from Fundação para a Ciência e Tecnologia, Portugal (contracts SFRH/BPD/46292/2008 and SFRH/BD/65962/2009 respectively).

\section{Conflict of interest disclosure}

The authors declare no competing financial interests. 


\section{Figure legends}

Figure 1. Antifungal resistance and tolerance in hematopoietic transplantation. Inflammatory dendritic cells (DCs), with some help from epithelial cells (ECs), provide antifungal resistance through the MyD88 pathway that restrains the fungal growth (via defensins) and leads to protective Th1/Th17 responses. In contrast, the coordinate action of ECs and tolerogenic DCs contribute to fungal tolerance through the TRIF pathway, which converges on IDO and expands Tbet $^{+}$Th1 and Foxp $3^{+}$Treg cells. 
Table 1. Relevant association studies of polymorphisms in pattern recognition receptors and susceptibility to fungal infections in HSCT recipients.

\begin{tabular}{|c|c|c|c|c|c|c|c|c|}
\hline Gene & SNPs & Source & Event & Effect & Cases (n) & Controls (n) & $P$ value & Ref. \\
\hline$T L R 1$ & $\mathrm{R} 80 \mathrm{~T}$ & Recipient & IA & $\mathrm{S}$ & 22 & 105 & 0.04 & [35] \\
\hline TLR1/TLR6 & N248S/S249P & Recipient & IA & $\mathrm{S}$ & 22 & 105 & 0.02 & [35] \\
\hline TLR4 & D299G/T399I & Donor & IA & S & 103 & 263 & 0.02 & [34] \\
\hline TLR4 & D299G/T399I & Donor & $\begin{array}{l}\text { Aspergillus } \\
\text { colonization }\end{array}$ & $\mathrm{S}$ & 58 & 51 & 0.003 & [36] \\
\hline TLR4 & D299G/T399I & Donor & IA & $\mathrm{R}$ & 34 & 24 & $0.03 \dagger$ & [36] \\
\hline DECTIN1 & Y238X & Recipient & $\begin{array}{l}\text { Candida } \\
\text { colonization }\end{array}$ & $\mathrm{S}$ & 46 & 78 & $<0.001$ & [39] \\
\hline DECTIN1 & Y238X & $\begin{array}{l}\text { Donor and } \\
\text { recipient }\end{array}$ & IA & S & 39 & 140 & 0.005 & [23] \\
\hline
\end{tabular}

$\dagger$ Resistance to IA was observed only when comparing IA vs. non-IA among pre-colonized patients with Aspergillus.

SNP: single nucleotide polymorphism; R: resistance; S: susceptibility; IA: invasive aspergillosis; HSCT: hematopoietic stem cell transplantation. 
Figure 1

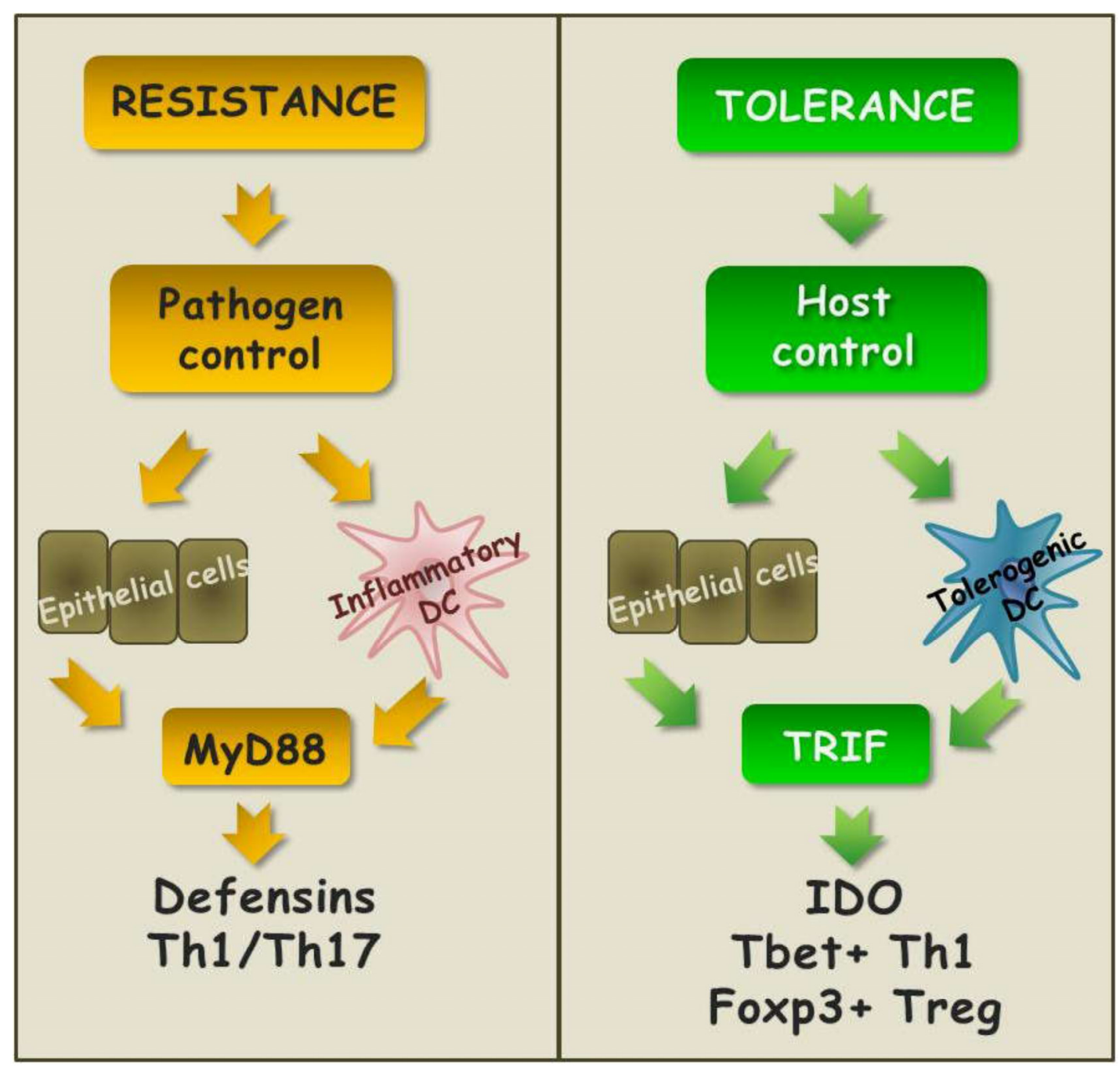




\section{References}

[1] Kontoyiannis DP, Marr KA, Park BJ, Alexander BD, Anaissie EJ, Walsh TJ, et al. Prospective surveillance for invasive fungal infections in hematopoietic stem cell transplant recipients, 2001-2006: overview of the Transplant-Associated Infection Surveillance Network (TRANSNET) Database. Clin Infect Dis. 2010;50:1091-100.

[2] Garcia-Vidal C, Upton A, Kirby KA, Marr KA. Epidemiology of invasive mold infections in allogeneic stem cell transplant recipients: biological risk factors for infection according to time after transplantation. Clin Infect Dis. 2008;47:1041-50.

[3] Romani L, Puccetti P. Controlling pathogenic inflammation to fungi. Expert Rev Anti Infect Ther. 2007;5:1007-17.

[4] Zelante T, De Luca A, D'Angelo C, Moretti S, Romani L. IL-17/Th17 in anti-fungal immunity: what's new? Eur J Immunol. 2009;39:645-8.

[5] Romani L, Zelante T, De Luca A, Fallarino F, Puccetti P. IL-17 and therapeutic kynurenines in pathogenic inflammation to fungi. J Immunol. 2008;180:5157-62.

[6] Curti A, Trabanelli S, Salvestrini V, Baccarani M, Lemoli RM. The role of indoleamine 2,3-dioxygenase in the induction of immune tolerance: focus on hematology. Blood. 2009;113:2394-401.

[7] Romani L, Fallarino F, De Luca A, Montagnoli C, D'Angelo C, Zelante T, et al. Defective tryptophan catabolism underlies inflammation in mouse chronic granulomatous disease.

Nature. 2008;451:211-5.

[8] Mitchell P, Afzali B, Lombardi G, Lechler RI. The T helper 17-regulatory T cell axis in transplant rejection and tolerance. Curr Opin Organ Transplant. 2009;14:326-31.

[9] Bonifazi P, D'Angelo C, Zagarella S, Zelante T, Bozza S, De Luca A, et al. Intranasally delivered siRNA targeting PI3K/Akt/mTOR inflammatory pathways protects from aspergillosis. Mucosal Immunol. 2010;3:193-205.

[10] Bozza S, Perruccio K, Montagnoli C, Gaziano R, Bellocchio S, Burchielli E, et al. A dendritic cell vaccine against invasive aspergillosis in allogeneic hematopoietic transplantation. Blood. 2003;102:3807-14.

[11] Bellocchio S, Montagnoli C, Bozza S, Gaziano R, Rossi G, Mambula SS, et al. The contribution of the Toll-like/IL-1 receptor superfamily to innate and adaptive immunity to fungal pathogens in vivo. J Immunol. 2004;172:3059-69.

[12] Mezger M, Kneitz S, Wozniok I, Kurzai O, Einsele H, Loeffler J. Proinflammatory response of immature human dendritic cells is mediated by dectin-1 after exposure to Aspergillus fumigatus germ tubes. J Infect Dis. 2008;197:924-31.

[13] Romani L, Bistoni F, Perruccio K, Montagnoli C, Gaziano R, Bozza S, et al. Thymosin alphal activates dendritic cell tryptophan catabolism and establishes a regulatory environment for balance of inflammation and tolerance. Blood. 2006;108:2265-74.

[14] Colonna M, Trinchieri G, Liu YJ. Plasmacytoid dendritic cells in immunity. Nat Immunol. 2004;5:1219-26.

[15] Naik SH, Proietto AI, Wilson NS, Dakic A, Schnorrer P, Fuchsberger M, et al. Cutting edge: generation of splenic CD8+ and CD8- dendritic cell equivalents in Fms-like tyrosine kinase 3 ligand bone marrow cultures. J Immunol. 2005;174:6592-7.

[16] Romani L, Bistoni F, Puccetti P. Fungi, dendritic cells and receptors: a host perspective of fungal virulence. Trends Microbiol. 2002;10:508-14.

[17] Bozza S, Clavaud C, Giovannini G, Fontaine T, Beauvais A, Sarfati J, et al. Immune sensing of Aspergillus fumigatus proteins, glycolipids, and polysaccharides and the impact on Th immunity and vaccination. J Immunol. 2009;183:2407-14. 
[18] Montagnoli C, Fallarino F, Gaziano R, Bozza S, Bellocchio S, Zelante T, et al. Immunity and tolerance to Aspergillus involve functionally distinct regulatory $\mathrm{T}$ cells and tryptophan catabolism. J Immunol. 2006;176:1712-23.

[19] Gribar SC, Richardson WM, Sodhi CP, Hackam DJ. No longer an innocent bystander: epithelial toll-like receptor signaling in the development of mucosal inflammation. Mol Med. 2008; 14:645-59.

[20] Desvignes L, Ernst JD. Interferon-gamma-responsive nonhematopoietic cells regulate the immune response to Mycobacterium tuberculosis. Immunity. 2009;31:974-85.

[21] Wasylnka JA, Moore MM. Aspergillus fumigatus conidia survive and germinate in acidic organelles of A549 epithelial cells. J Cell Sci. 2003;116:1579-87.

[22] Balloy V, Sallenave JM, Wu Y, Touqui L, Latge JP, Si-Tahar M, et al. Aspergillus fumigatus-induced interleukin-8 synthesis by respiratory epithelial cells is controlled by the phosphatidylinositol 3-kinase, p38 MAPK, and ERK1/2 pathways and not by the toll-like receptor-MyD88 pathway. J Biol Chem. 2008;283:30513-21.

[23] Cunha C, Di Ianni M, Bozza S, Giovannini G, Zagarella S, Zelante T, et al. Dectin-1 Y238X polymorphism associates with susceptibility to invasive aspergillosis in hematopoietic transplantation through impairment of both recipient- and donor-dependent mechanisms of antifungal immunity. Blood. 2010.

[24] De Luca A, Bozza S, Zelante T, Zagarella S, D’Angelo C, Perruccio K, et al. Nonhematopoietic cells contribute to protective tolerance to Aspergillus fumigatus via a TRIF pathway converging on IDO. Cell Mol Immunol. 2010.

[25] Zelante T, De Luca A, Bonifazi P, Montagnoli C, Bozza S, Moretti S, et al. IL-23 and the Th17 pathway promote inflammation and impair antifungal immune resistance. Eur $\mathrm{J}$ Immunol. 2007;37:2695-706.

[26] Paveglio SA, Allard J, Foster Hodgkins SR, Ather J, Bevelander M, Mayette Campbell J, et al. Airway Epithelial Indoleamine 2,3-Dioxygenase Inhibits CD4+ T Cells During Aspergillus fumigatus Antigen Exposure. Am J Respir Cell Mol Biol. 2010.

[27] Sha Q, Truong-Tran AQ, Plitt JR, Beck LA, Schleimer RP. Activation of airway epithelial cells by toll-like receptor agonists. Am J Respir Cell Mol Biol. 2004;31:358-64. [28] Mayer AK, Bartz H, Fey F, Schmidt LM, Dalpke AH. Airway epithelial cells modify immune responses by inducing an anti-inflammatory microenvironment. Eur J Immunol. 2008;38:1689-99.

[29] Bretz C, Gersuk G, Knoblaugh S, Chaudhary N, Randolph-Habecker J, Hackman RC, et al. MyD88 signaling contributes to early pulmonary responses to Aspergillus fumigatus. Infect Immun. 2008;76:952-8.

[30] De Luca A, Montagnoli C, Zelante T, Bonifazi P, Bozza S, Moretti S, et al. Functional yet balanced reactivity to Candida albicans requires TRIF, MyD88, and IDO-dependent inhibition of Rorc. J Immunol. 2007;179:5999-6008.

[31] Carvalho A, Cunha C, Pasqualotto AC, Pitzurra L, Denning DW, Romani L. Genetic variability of innate immunity impacts human susceptibility to fungal diseases. Int J Infect Dis. 2010;14:e460-8.

[32] Arbour NC, Lorenz E, Schutte BC, Zabner J, Kline JN, Jones M, et al. TLR4 mutations are associated with endotoxin hyporesponsiveness in humans. Nat Genet. 2000;25:187-91.

[33] Carvalho A, Pasqualotto AC, Pitzurra L, Romani L, Denning DW, Rodrigues F.

Polymorphisms in toll-like receptor genes and susceptibility to pulmonary aspergillosis. J Infect Dis. 2008;197:618-21.

[34] Bochud PY, Chien JW, Marr KA, Leisenring WM, Upton A, Janer M, et al. Toll-like receptor 4 polymorphisms and aspergillosis in stem-cell transplantation. N Engl J Med. 2008;359:1766-77. 
[35] Kesh S, Mensah NY, Peterlongo P, Jaffe D, Hsu K, M VDB, et al. TLR1 and TLR6 polymorphisms are associated with susceptibility to invasive aspergillosis after allogeneic stem cell transplantation. Ann N Y Acad Sci. 2005;1062:95-103.

[36] Carvalho A, Cunha C, Carotti A, Aloisi T, Guarrera O, Di Ianni M, et al. Polymorphisms in Toll-like receptor genes and susceptibility to infections in allogeneic stem cell transplantation. Exp Hematol. 2009;37:1022-9.

[37] Kiechl S, Lorenz E, Reindl M, Wiedermann CJ, Oberhollenzer F, Bonora E, et al. Tolllike receptor 4 polymorphisms and atherogenesis. N Engl J Med. 2002;347:185-92.

[38] Ferwerda B, Ferwerda G, Plantinga TS, Willment JA, van Spriel AB, Venselaar H, et al. Human dectin-1 deficiency and mucocutaneous fungal infections. N Engl J Med. 2009;361:1760-7.

[39] Plantinga TS, van der Velden WJ, Ferwerda B, van Spriel AB, Adema G, Feuth T, et al. Early stop polymorphism in human DECTIN-1 is associated with increased candida colonization in hematopoietic stem cell transplant recipients. Clin Infect Dis. 2009;49:724-32. [40] Werner JL, Metz AE, Horn D, Schoeb TR, Hewitt MM, Schwiebert LM, et al. Requisite role for the dectin-1 beta-glucan receptor in pulmonary defense against Aspergillus fumigatus. J Immunol. 2009;182:4938-46.

[41] Carvalho A, Cunha C, Di Ianni M, Pitzurra L, Aloisi T, Falzetti F, et al. Prognostic significance of genetic variants in the IL-23/Th17 pathway for the outcome of T cell-depleted allogeneic stem cell transplantation. Bone Marrow Transplant. 2010.

[42] Elmaagacli AH, Koldehoff M, Landt O, Beelen DW. Relation of an interleukin-23 receptor gene polymorphism to graft-versus-host disease after hematopoietic-cell transplantation. Bone Marrow Transplant. 2008;41:821-6.

[43] Meglio PD, Cesare AD, Laggner U, Chu C-C, Peris K, Nestle F. F.15. A Gene-toFunction Analysis of IL-23R Arg381Gln Polymorphism Reveals Impaired IL-17A Production in Th17 Cells. Clinical Immunology. 2009;131:S97-S. 